\title{
The scientific social network
}

\section{A joint statement from 17 funding agencies urges biomedical researchers to openly share data obtained from population-based studies. Although this will foster more collaboration, new web technologies need to be harnessed, and the attribution of credit must change to facilitate this transition.}

$\mathbf{T}$ he widespread adoption of social networks, wikis and blogs has fostered an environment of sharing and collaboration. For scientists, this provides the opportunity to generate and curate content more seamlessly and dynamically than ever before. Web 2.0 applications targeted at researchers are gaining in popularity but need to be adopted more widely to stimulate a more collaborative and open research environment.

Researchers in genetics and the physical sciences have taken the lead in allowing public access to research by depositing results in databases and through the use of preprint servers such as arXiv (http:// arxiv.org/) and Nature Precedings (http://precedings.nature.com/). Following their example, a recent joint statement (Lancet, doi:10.1016/ S0140-6736(10)62234-9, 2011) from 17 funding agencies including the Wellcome Trust and the US National Institutes of Health urges scientists to "increase the availability... of the research data we fund that is collected from populations for the purpose of health research." The statement calls for primary data sets to be deposited in central databases and made available to the global research community for analysis.

Data sharing has been implemented successfully in scattered initiatives in the biomedical field, such as the Alzheimer's Disease Neuroimaging Initiative. From 2004 to 2009, researchers obtained longitudinal data, which are readily available to researchers who apply for access, on 819 subjects aimed at identifying biomarkers of disease initiation and progression (Alzheimers Dement. 6, 202-211, 2010). To date, 242 papers have been published that use this data set, and the project has been extended for an additional five years to study antecedents of disease. Whereas this example demonstrates the utility of data sharing, the quantity and complexity of the data being generated and deposited online requires new tools to organize and highlight these data and to encourage researchers from diverse areas to work together in analyzing them.

Social networking sites hold considerable promise in fostering productive research collaborations. The site ResearchGATE (http:// www.researchgate.net/) hosts a community of 700,000 scientists, allowing users to create profiles, identify collaborators, critique and highlight articles and continue discussions after a scientific meeting has adjourned. One advantage of such a website is the ability to crowd-source: individuals post problems or queries on an open forum, relying on the expertise of the community to provide answers and promote an exchange of ideas.

In this vein, Nature Publishing Group has partnered with InnoCentive to develop Nature Innovation Pavilion (http://www2. innocentive.com/pavilion/nature/), a platform for harnessing the power of crowd sourcing to solve research problems. Anyone (termed 'Seekers') can post research and development problems or projects on an open forum, allowing individuals (termed 'Solvers') to submit possible solutions. Once the Seeker picks a solution, the Solver receives a predetermined cash reward. From 2001 to 2004, 166 problems from 26 firms were posted at InnoCentive. From a network of 80,000 scientists, 993 solutions were submitted, leading to 49 challenges solved. In fact, Solvers with technical expertise that was complementary to those posed in the challenge were more likely to submit successful solutions (Organ. Sci. 21, 1016-1033, 2010). This model of problem solving points to the usefulness of mass collaboration for providing novel solutions to complex problems and, in the future, may provide another form of funding for smaller labs with specialized expertise.

Large communities of scientists can also be called upon to curate wikis. Given the speed at which new studies are published, wiki-style community collaborations allow consolidation and annotation of these data into a central resource that can constantly be updated. The popularity of several wikis, including OpenWetWare (http://openwetware. org/), which provides a database of protocols and allows users to generate a virtual lab notebook, and WikiPathways (http://www.wikipathways. org/), a database of biological pathways and networks, points to the usefulness of this format for scientific discourse.

Increased data sharing and the growing prominence of communitycurated sites may propel a rethinking of how credit is assigned, funding is distributed and science is reported. A recent survey of UK academics cites current reward structures and recognition as one reason contributing to the relatively modest adoption of these technologies amongst researchers (Phil. Trans. R. Soc. A 368, 4039-4056, 2010). In the Lancet statement the 17 funding organizations recognize these hurdles and state that the attribution of credit needs to be altered to recognize collaborative efforts and other forms of scholarly communication in addition to peer-reviewed publications. Once these obstacles are cleared, these resources may become mainstream tools, ushering in a new era of mass collaboration among biomedical researchers. 